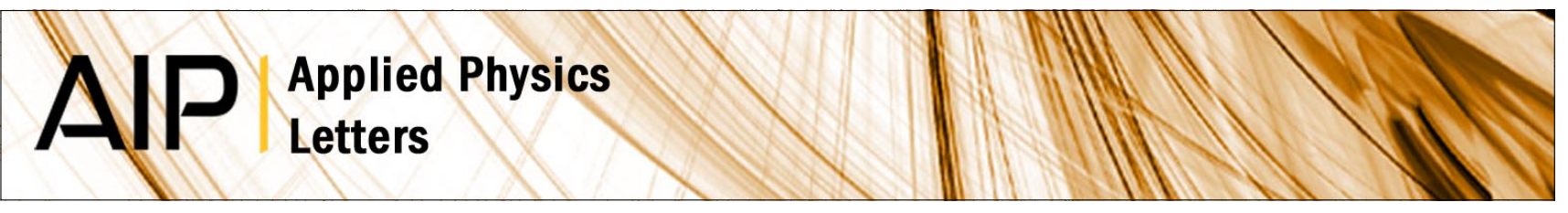

\title{
Experimental cross-polarization detection of coupling far-field light to highly confined plasmonic gap modes via nanoantennas
}

J. Wen, P. Banzer, A. Kriesch, D. Ploss, B. Schmauss et al.

Citation: Appl. Phys. Lett. 98, 101109 (2011); doi: 10.1063/1.3564904

View online: http://dx.doi.org/10.1063/1.3564904

View Table of Contents: http://apl.aip.org/resource/1/APPLAB/v98/i10

Published by the American Institute of Physics.

\section{Related Articles}

Localized surface plasmon resonances in highly doped semiconductors nanostructures Appl. Phys. Lett. 101, 161113 (2012)

Plasmonic coupling effect between two gold nanospheres for efficient second-harmonic generation J. Appl. Phys. 112, 083102 (2012)

Controlled spatial switching and routing of surface plasmons in designed single-crystalline gold nanostructures Appl. Phys. Lett. 101, 141114 (2012)

Negative and positive photoconductivity modulated by light wavelengths in carbon nanotube film Appl. Phys. Lett. 101, 123117 (2012)

Polarizability of supported metal nanoparticles: Mehler-Fock approach

J. Appl. Phys. 112, 064312 (2012)

\section{Additional information on Appl. Phys. Lett.}

Journal Homepage: http://apl.aip.org/

Journal Information: http://apl.aip.org/about/about_the_journal

Top downloads: http://apl.aip.org/features/most_downloaded

Information for Authors: http://apl.aip.org/authors

\section{ADVERTISEMENT}
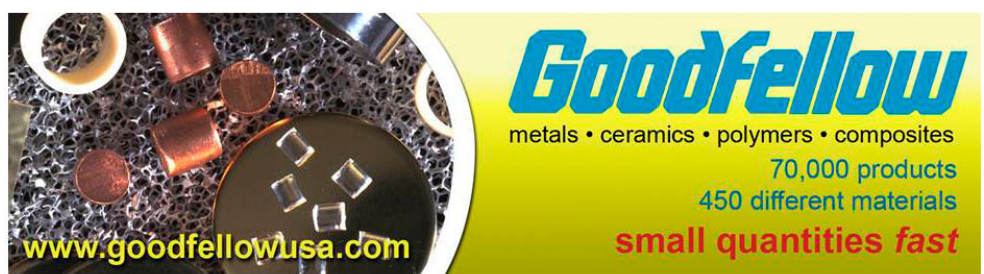


\title{
Experimental cross-polarization detection of coupling far-field light to highly confined plasmonic gap modes via nanoantennas
}

\author{
J. Wen, ${ }^{1, a)}$ P. Banzer, ${ }^{1}$ A. Kriesch, ${ }^{1}$ D. Ploss, ${ }^{1}$ B. Schmauss, ${ }^{2}$ and U. Peschel ${ }^{1}$ \\ ${ }^{1}$ Institute of Optics, Information and Photonics and Max Planck Institute for the Science of Light, \\ Günther-Scharowsky-Str. 1, Building 24, 91058 Erlangen, Germany \\ ${ }^{2}$ Chair for High Frequency Technology, University of Erlangen-Nuremberg, Cauerstr. 9, 91058 Erlangen, \\ Germany
}

(Received 20 December 2010; accepted 14 February 2011; published online 10 March 2011)

\begin{abstract}
We experimentally demonstrate the coupling of far-field light to highly confined plasmonic gap modes via connected nanoantennas. The excitation of plasmonic gap modes is shown to depend on the polarization, position, and wavelength of the incident beam. Far-field measurements performed in crossed polarization allow for the detection of extremely weak signals re-emitted from gap waveguides and can increase the signal-to-noise ratio dramatically. (C) 2011 American Institute of Physics. [doi:10.1063/1.3564904]
\end{abstract}

Surface plasmon polaritons (SPPs) are highly confined electromagnetic waves on metal-dielectric interfaces which couple to collective oscillations of the electron gas. Various plasmonic waveguide modes arise from the coupling of SPPs at the interfaces of metals and dielectrics. ${ }^{1-6}$ Bozhevolnyi et $a l^{7}$ reported a channel waveguide mode with a width of $1.1 \mu \mathrm{m}$ and a propagation length of $100 \mu \mathrm{m}$. However, most of the experimentally realized plasmonic modes ${ }^{1,7}$ have confinements in the micrometer range and do not reach deeply subwavelength dimensions. Thus they do not have obvious advantages compared to conventional dielectric waveguides. In contrast, a true deeply subwavelength gap between two metal surfaces can support an extremely confined mode, but such a compact geometry is more challenging to operate in terms of excitation and detection compared to other structures. Verhagen et al. ${ }^{2}$ reported the near-field detection of metal-insulator-metal modes in multilayer structures which showed high confinement perpendicular to the sample surface. Usually, standard phase-matching techniques are employed to excite SPPs, such as Kretschmann, Otto, and grating configurations. However, other excitation techniques need to be developed for launching SPPs in deeply subwavelength dimensions. For the excitation of lessconfined stripe wave guide modes, generators like arcshaped nanodots and slits were reported by Imre et al. $^{3}$ and Nomura et al. ${ }^{4}$ Recently, two-dimensional, highly confined gap modes were experimentally realized by Chen et al. ${ }^{5}$ and Han et al. ${ }^{6}$ They showed the integration of slot waveguides with silicon dielectric waveguides via tapered couplers. However, the aforementioned excitation schemes often lack the compactness that is required for integration into subwavelength, plasmonic circuitry. Nanoantennas are highly compact and were proposed as a kind of nanocoupler to couple far-field light to two-dimensional, highly confined gap modes. ${ }^{8,9}$ Recently, the idea of optical wireless interconnects via nanoantennas was theoretically proposed by Alù and Engheta. ${ }^{10}$ Thus loading the nanoantennas with plasmonic waveguides is the first step toward optical wireless communication analogous to the ubiquitous loading of radio antennas with coaxial cables in the regime of radio fre-

${ }^{a)}$ Electronic mail: jing.wen@mpl.mpg.de. quency. In this paper, we experimentally demonstrate the excitation of a gap mode enhanced by a nanoantenna and investigate its spatial and spectral dependence on the antenna by cross-polarization detection (see Fig. 1). We prove that optical antennas can potentially be used for selective excitation of highly confined, plasmonic waveguide modes in further integrated plasmonic chips.

For the experiments a 100-nm-thick silver layer with a surface roughness of around $1 \mathrm{~nm}$ was deposited on a glass substrate using a magnetron sputtering machine. A chromium layer with a thickness of 1-2 nm was used as a wetting layer to improve the quality of the silver layer. Afterwards 80 100 -nm-wide grooves were structured into the silver layer using a focused ion beam (FIB) milling system. A scanning electron microscope (SEM) image of a cross section of the waveguide and the corresponding numerically calculated mode profile (via finite-element method, FEM) of the electric field are given in Fig. 2(a). Figure 2(b) shows an SEM image of the measured structure containing a bent-gap waveguide with a receiving antenna. Figure 2(c) shows the same structure but includes an additional transmitting antenna at the other end of the bent waveguide. The gap width is $80 \mathrm{~nm}$. The optimized total length of the antenna is designed for a resonance at around $\lambda=1.5 \mu \mathrm{m}$.

The configuration of the experimental setup is shown in Fig. 1. For excitation, a linearly polarized Gaussian beam (polarization parallel to the antenna) was focused onto the

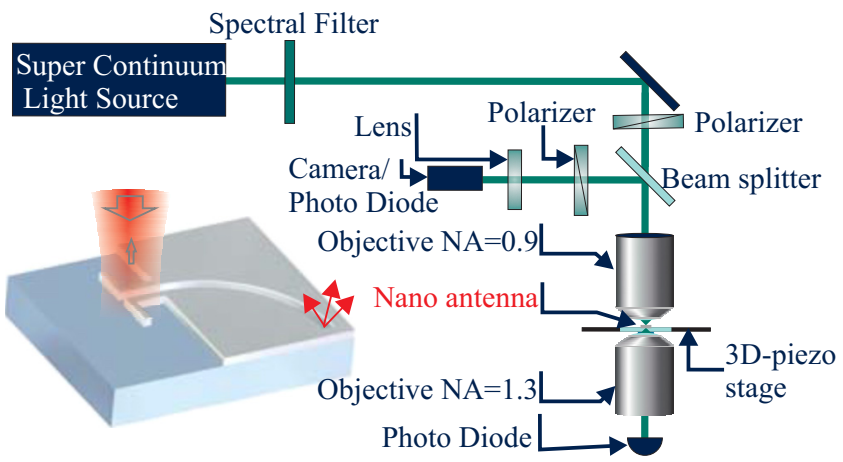

FIG. 1. (Color online) The configuration of the experimental setup and the schematic view of the measurement. 

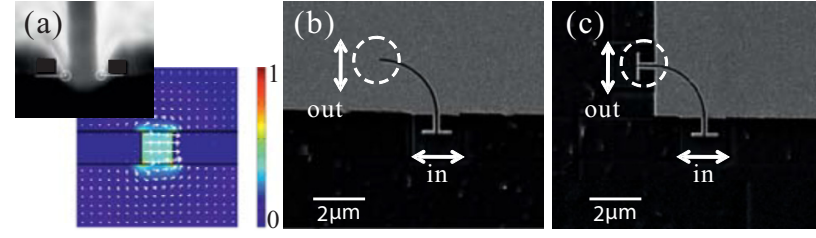

Without crossed polarizer With crossed polarizer With crossed polarize
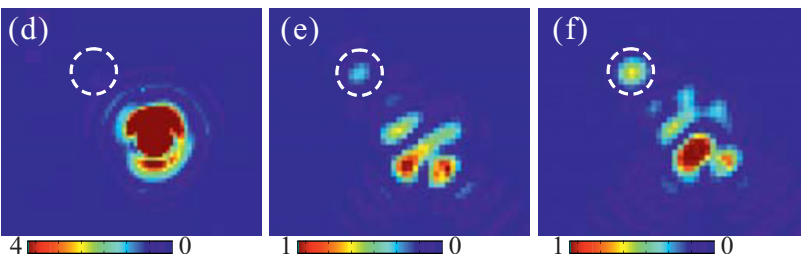

FIG. 2. (Color online) (a) SEM image of a cross section of the waveguide and the corresponding mode profile of the electric field (FEM). Optical excitation of gap waveguide modes via nanoantennas and detection of the light backscattered by the waveguide end (b)-(e) or an emitting antenna (c) and (f). Panels [(b) and (c)]: SEM images. White arrows indicate the polarization direction of the incident beam and the direction of the crossed polarizer in front of the camera. Panels (d)-(f): optical reflection camera images with (e) and (f) and without (d) a crossed polarizer in front of the charge-coupled device camera. $(\lambda=1.5 \mu \mathrm{m})$. The residual spatial intensity pattern of the optical beam in the crossed polarization scheme shown in panels (e) and (f) was explained by Ref. 11.

substrate surface. Due to strong back reflection of the incident beam, it is hard to investigate the field in the waveguide unless its direction of polarization is turned so that it becomes visible in the cross-polarized direction. Since the polarization of gap modes is always perpendicular to the tangent of the waveguide, the polarization of the electric fields at the waveguide end can be turned by $90^{\circ}$ via a waveguide bend. Thus, the dominant part of the back reflection of the incident beam was filtered out whereas the scattered light from the very end of the waveguide was detected. For comparison, Fig. 2(d) shows the reflection image of the sample surface recorded by a camera without a crossed polarizer in front of the camera. In Figs. 2(e) and 2(f) two cases with a crossed polarizer in front of the camera are shown. The large bright spot in Fig. 2(d) is caused by the reflection of the focused beam by the nanoantenna. On the upper-left side of the reflected beam, there is only a tiny weak spot with strong background noise. However, for crossed-polarizer detection, a small but clear spot can be observed [see spots inside the white-dashed circles in Figs. 2(e) and 2(f)]. This demonstrates that the focused optical beam is coupled to the highly confined mode of the plasmonic waveguide via the resonant receiving antenna. Apparently, cross-polarization detection increases the signal-to-noise ratio (SNR) dramatically. In addition, this proves that we have high transmission through bends with micrometer-sized radii. The emission intensity with a second transmitting antenna [see Figs. 2(c) and 2(f)] is enhanced by at least a factor of 2 compared to the case with just an open end [see Figs. 2(b) and 2(e)]. The observed scattering at the end of the waveguide weakens and shifts as expected if the sample is moved relative to the optical beam. For an excitation with an incident polarization perpendicular to the long axis of the antenna, at a nonresonant wavelength or without a receiving antenna (reflection images not presented here), no light is observed at the end of the waveguide.

Figure 3 shows scans measured in transmission and reflection at the resonant wavelength of $1.5 \mu \mathrm{m}$ in (a) and (b)

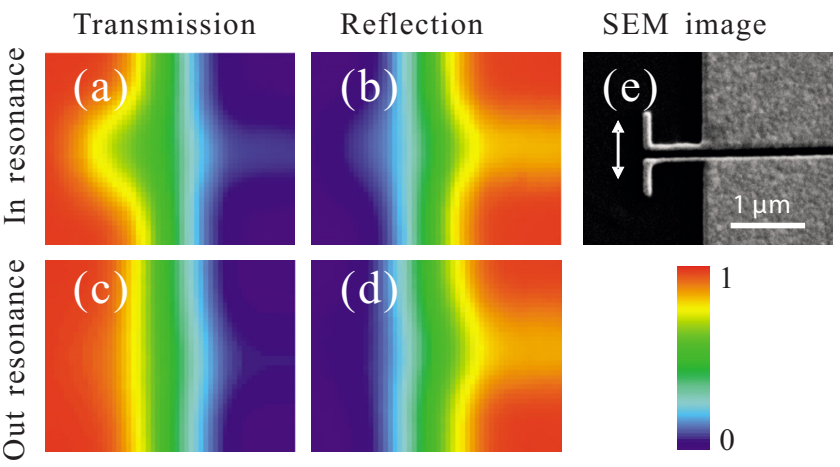

FIG. 3. (Color online) Scanning measurements in transmission (a) and reflection (b) at resonant wavelength of $1.5 \mu \mathrm{m}$. Scanning measurements in transmission (c) and reflection (d) at nonresonant wavelength of $1 \mu \mathrm{m}$. (e) SEM image of the corresponding scanning area including the antenna and part of the straight waveguide. Polarization of the incident beam (white arrow) is parallel to the antenna axis. All scans are acquired without a detector polarizer

and at a nonresonant wavelength of $1 \mu \mathrm{m}$ in (c) and (d). For each scan, the incident polarization was parallel to the antenna. No polarizer was situated in front of the detecting photo diode. Each scanning pixel value corresponds to the normalized transmission and reflection measured for a certain position of the focused beam relative to the sample. The sample was scanned through the beam using a defined step size. Transmission and reflection intensities were measured for every position. All presented scans were normalized to the same level. The transmission for resonant excitation of the antenna is lower [yellow color in Fig. 3(a)] than the value at the nonresonant wavelength [red color in Fig. 3(c)]. In the same situation, the reflection does not vary much [see Figs. 3(b) and 3(d)]. This already hints that light is coupled into the waveguide for resonant excitation. We also measured the transmission and reflection spectra of the antenna for incident wavelengths ranging from 1 to $1.7 \mu \mathrm{m}$ (see Fig. 4). The transmission drops by about $10 \%$ close to $\lambda=1.5 \mu \mathrm{m}$ but the reflection stays almost constant for all measured wavelengths. A quite intuitive explanation suggests that, at resonance, energy is coupled to the waveguide mode and results in a reduced transmission but leaves the reflection almost unaffected. For comparison, we simulated the coupling efficiency using the COMSOL MULTIPHYSICS commercial FEM

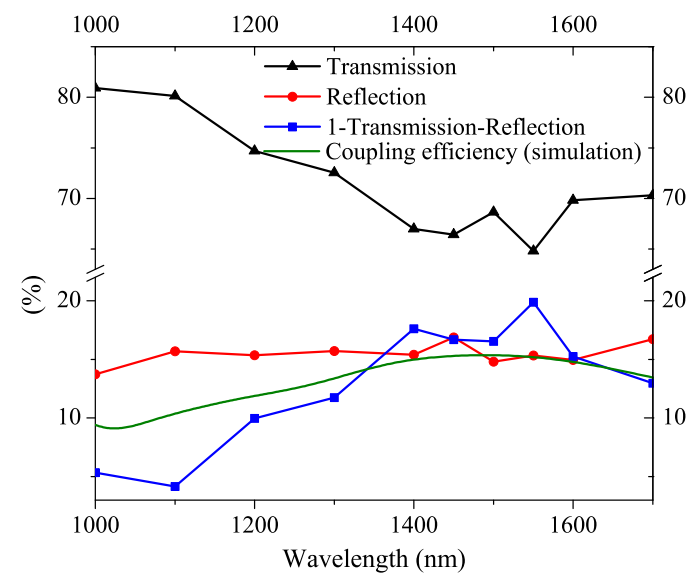

FIG. 4. (Color online) Experimentally measured spectra in transmission and reflection (normalized). The simulated coupling efficiency of the antennawaveguide system is shown in the wavelength range between 1 and $1.7 \mu \mathrm{m}$. 


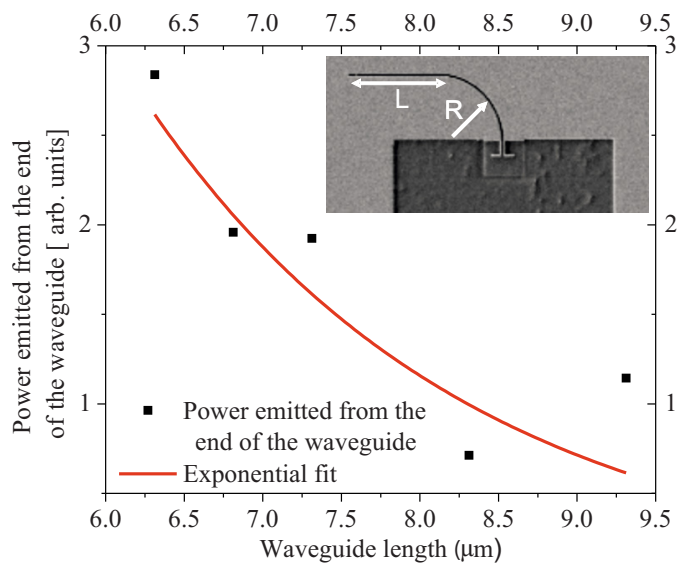

FIG. 5. (Color online) Power emitted from the end of the waveguide vs total waveguide length, which is fit exponentially (solid line). Inset: SEM image of one measured sample. The radius of the bent part (R) is $3 \mu \mathrm{m}$. The length of the straight part (L) varies from 1 to $4 \mu \mathrm{m}$.

package. In order to get a continuous spectrum, the transient analysis module was used. An excitation port which generated an ultrashort electric pulse (spectral width $1.8 \mu \mathrm{m}$ and central wavelength $1.54 \mu \mathrm{m}$ ) was set at the end of the waveguide far away from the antenna. The detection port with an area of $1.5 \times 1.5 \mu^{2}(40 \times 40$ grid $)$ was placed $200 \mathrm{~nm}$ above the antenna in air. The powerflow at each point of the detection port in the frequency domain was calculated from the Fourier transform of the electric and magnetic fields. The powerflow was then integrated over the detection port area and normalized to the value on the port at the connection between the antenna and the waveguide with an area of $200 \times 200 \mathrm{~nm}^{2}$. This is to exclude the dispersion effect of the waveguide. Suppose the whole process is reciprocal, we thus obtain the maximum coupling efficiency for an excitation from air. The simulated coupling efficiency shows a maximum around $1.5 \mu \mathrm{m}$, matching the experimental resonance quite well. Experiments and simulations suggest that about $15 \%$ of the beam's energy is coupled to the waveguide. It should be noted that the calculated coupling efficiency for an excitation from underneath through the substrate is 1.3 times higher.

Since we were able to control the waveguide excitation, we also determined the decay length of gap modes with a width of $80 \mathrm{~nm}$ at $\lambda=1.5 \mu \mathrm{m}$. As shown in Fig. 5, the radius of the bent part (R) is fixed to $3 \mu \mathrm{m}$, and the length of the straight part (L) varies from 1 to $4 \mu \mathrm{m}$. The emission from the waveguide end was used for detection. The light emitted by the waveguide end was extracted and normalized to the power of the optical beam focused on the pure glass substrate. The coupling efficiency of the antenna on each measured sample was maximized by adjusting the position of the nanoantenna relative to the focused beam using a piezostage. By fitting an exponential decay function to the derived data, the decay length was found to be $2.1 \pm 0.8 \mu \mathrm{m}$. The rather low value of the decay length might be due to the fact that the fabricated waveguide width of $80 \mathrm{~nm}$ is expected to be near cutoff. Also the losses of sputtered silver layers seem to be much higher than the values for silver ${ }^{12}$ used in the simulation. Another reason for the low decay length might be that the FIB structuring process introduces roughness on the sidewalls of the gap, which results in additional scattering losses.

In summary, the excitation and detection of plasmonic gap modes has been demonstrated. By using a crossedpolarizer detection scheme that requires bent waveguides, the SNR was dramatically improved. The excitation of gap modes was shown to be sensitive to the wavelength and position of the excitation beam. The measured sum of the coupling efficiency and antenna absorption reached up to $20 \%$ compared to the simulated optimum coupling efficiency of $15 \%$.

The author acknowledges the International Max-Planck Research School (IMPRS) for Optics and Imaging, the Cluster of Excellence for "Engineering of Advanced Materials" (EAM) at the University of Erlangen-Nuremberg, and Erlangen Graduate School in Advanced Optical Technologies (SAOT).

${ }^{1}$ S. A. Maier, M. D. Friedman, P. E. Barclay, and O. Painter, Appl. Phys. Lett. 86, 071103 (2005).

${ }^{2}$ E. Verhagen, J. A. Dionne, L. K. Kuipers, H. A. Atwater, and A. Polman, Nano Lett. 8, 2925 (2008).

${ }^{3}$ A. Imre, V. K. V. Vlasov, J. Pearson, J. M. Hiller, and U. Welp, Appl. Phys. Lett. 91, 083115 (2007).

${ }^{4}$ W. Nomura, M. Ohtsu, and T. Yatsui, Appl. Phys. Lett. 86, 181108 (2005).

${ }^{5}$ L. Chen, J. Shakya, and M. Lipson, Opt. Lett. 31, 2133 (2006).

${ }^{6}$ Z. Han, A. Y. Elezzabi, and V. Van, Opt. Lett. 35, 502 (2010).

${ }^{7}$ S. I. Bozhevolnyi, V. S. Volkov, E. Devaux, J.-Y. Laluet, and T. W. Ebbesen, Nature (London) 440, 508 (2006).

${ }^{8}$ J. Wen, S. Romanov, and U. Peschel, Opt. Express 17, 5925 (2009).

${ }^{9}$ J.-S. Huang, T. Feichtner, P. Biagioni, and B. Hecht, Nano Lett. 9, 1897 (2009)

${ }^{10}$ A. Alù and N. Engheta, Phys. Rev. Lett. 104, 213902 (2010).

${ }^{11}$ M. Mansuripur, J. Opt. Soc. Am. A 3, 2086 (1986).

${ }^{12}$ E. D. Palik, Handbook of Optical Constants (Academic, New York, 1985). 